\title{
Review of Green Synthesis and Antimicrobial Efficacy of Copper and Nickel Nanoparticles
}

\author{
Ahtesham Ahmad Shad ${ }^{1 *}$ and Wajahat Ahmed Shad ${ }^{2}$ \\ ${ }^{1}$ Institute of Microbiology, University of Agriculture Faisalabad, Pakistan \\ ${ }^{2}$ Department of Biochemistry, University of Agriculture Faisalabad, Pakistan \\ *Corresponding author: Ahtesham Ahmad Shad, University of Agriculture Faisalabad, Pakistan. \\ To Cite This Article: Ahtesham Ahmad Shad. Review of Green Synthesis and Antimicrobial Efficacy of Copper and Nickel Nanoparticles. Am J \\ Biomed Sci \& Res. 2019 - 3(6). AJBSR.MS.ID.000721. DOI: 10.34297/AJBSR.2019.03.000721
}

Received: June 24, 2019 | Published: July 09, 2019

\begin{abstract}
Nanotechnology is the field of significant importance and exhibit versatility in approaching other sciences at micro to macro levels. The presence of nanoparticles in natural environment has gained special attention recently in medical world. Because of smaller size, inorganic and organic nature, optical phenomenon, physical and biochemical properties in relation with size to volume the nanoparticles are applicable in various disciplines and sciences. Moreover, due to major threat of prevailing antimicrobial resistance (AMR) and slow progress in developing new drugs, the need of time also provoked these nano-technological approaches due to their antibacterial activity. The various kinds of nano-particles have been extracted and prepared from various methods i.e. gold, silver etc. This review aimed to highlight green synthesis phenomenon in preparation of copper $(\mathrm{Cu})$ and nickel (Ni) nanoparticles as few data is available. Moreover, antibacterial efficacies of these nanoparticles are also explained to give a better look in understanding and expanding the knowledge accompanied with already described other nanoparticles.
\end{abstract}

Keywords: Nano-particles; Green synthesis; AMR

\section{Introduction}

Nanotechnology is the field of science relating to preparation, extraction and characterization of very small size particles ranged in 1-100nm [1]. These very small particles are manipulated in different disciplines according to their properties elasticity as behave differently in bulk or in particle form due to significant relation of size and volume. The versatility in behavior would likely be due to large surface area of these smaller nanoparticles and different quantum mechanics in comparison to the particles in bulk $[2,3]$. There are generally two core methods have been developed to prepare and utilize these nanoparticles according to desire need and requirement i.e. top down and bottom up methods [4]. Moreover, these two approaches for obtaining the nanoparticles included various techniques as shown in Figure 1. The parameters for synthesizing nano-particles like shape and size can be modified according to need by modulating reaction conditions and chemical ingredients. But these chemically synthesized and modified nanoparticles have challenges and limitations like instability, toxicity, expensiveness, accumulation in devices and recycling capacities. Meanwhile due to these hurdles and problems science technology improvement leads to emerge a new gate to synthesize nanoparticles that is "Green-Synthesis". The green synthesis approach has significantly gained interest to develop and prepare nano-particles because of their eco-friendly feature. The green synthesis method implied the usage of regulatory, controlling, cleanup and remediation steps. Green synthesis technique minimizes the waste, reduces the pollutants and their derivatives and also is non-toxic as well [5]. The green synthesis techniques to synthesize nano-particles are based on living entities and biological precursors produced during chemical reactions. The various biologically important chemicals and by-products are capable enough to reduce the metallic salts into their metallic nano-particles upon reactivity. The sources of green synthesis are represented in Figure 2.

So, this attempt is made to highlight the green synthesis of Copper and Nickle nano-particles as very little data is available as compare to silver and gold nano-particles. The descriptive approach regarding the characteristics and features of Copper $(\mathrm{Cu})$ and Nickle (Ni) nano-particles using green synthesis method is explained to better understanding of these nano-particles. Moreover, more specifically the antimicrobial efficacies of these $\mathrm{Cu}$ and $\mathrm{Ni}$ nanoparticles are also discussed to help researchers and other health care workers looking for possible solutions to tackle the burden 
of antimicrobial resistance as drug resistance has prevailed all across the globe and assuming $\mathrm{Cu}$ and $\mathrm{Ni}$ nanoparticles as potent alternative therapeutic agents as treatment option.

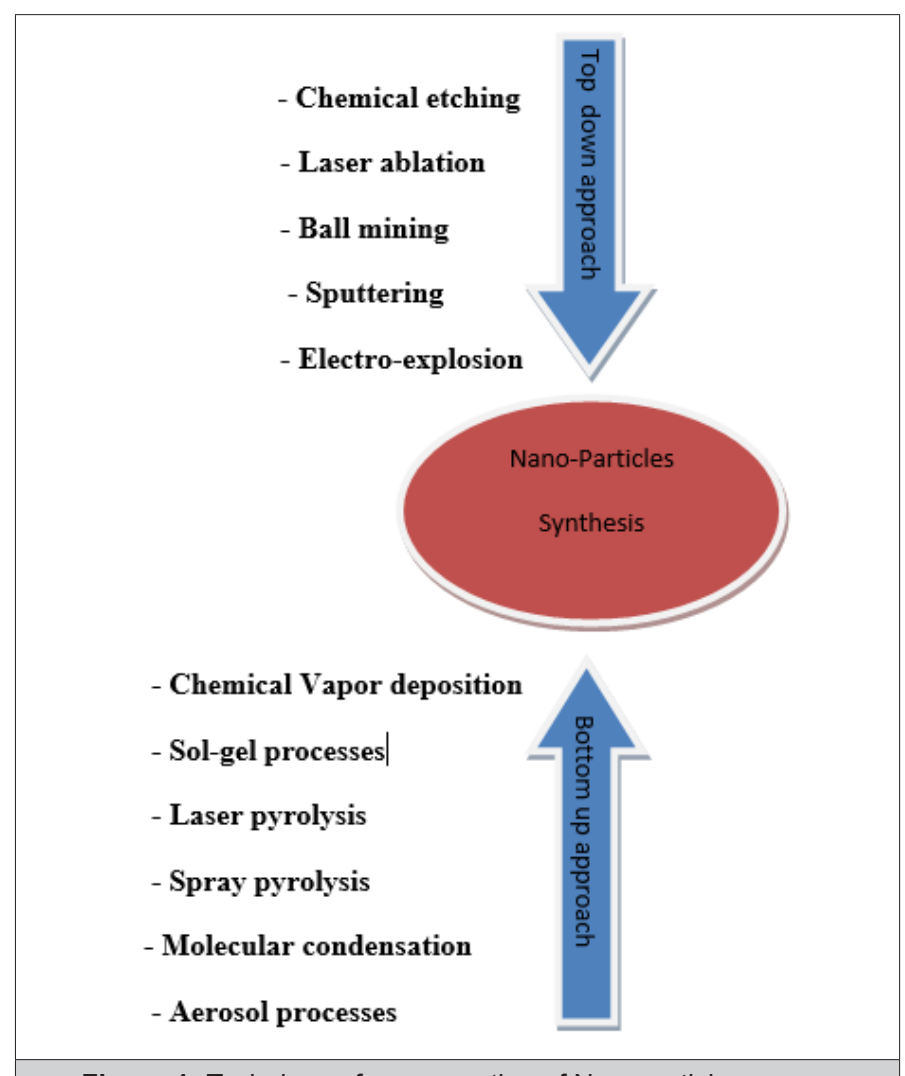

Figure 1: Techniques for preparation of Nanoparticles.

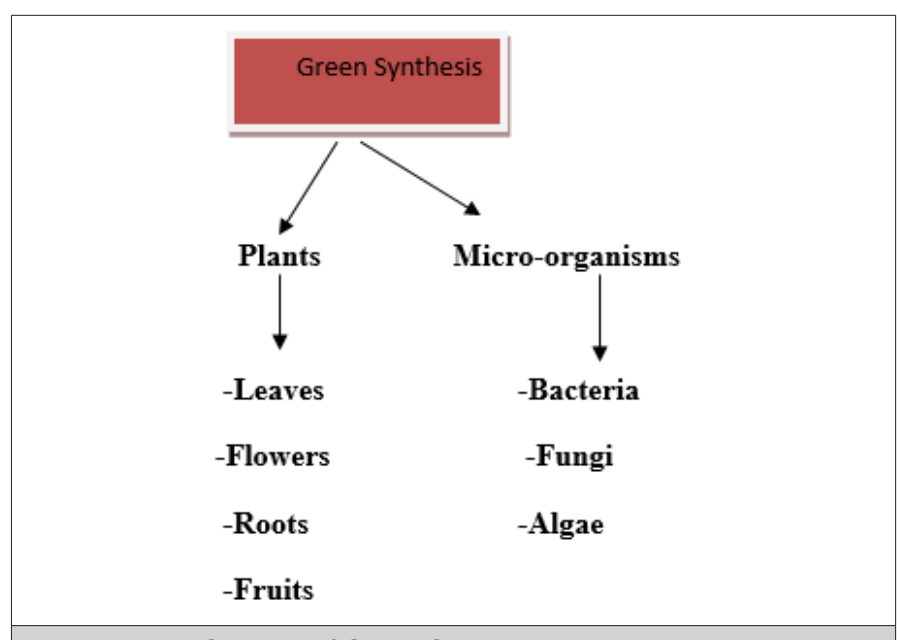

Figure 2: Sources of Green Synthesis.

\section{Green Synthesis of Copper $(\mathrm{Cu})$ Nano-particles}

In past, scientists and researchers made their very best to find new ways to synthesize metal NPs. One of the valuable and attention seeking approach is "Green synthesis" [6,7]. There was also focused on microorganisms for getting nano-particles accompanied with plants and their extracts [8,9]. Plants have various advantages over other described biological methods as absence of pathogenecity. Copper has also several distinct features in synthesizing nano-particles as at room temperature its can easily be oxidized [10]. While in comparison with silver and gold nano- particles, they have oxidation higher to $\mathrm{Cu}$. Moreover the copper is more promising material as because of its abundance in nature and low cost. There are several capping agents are applied to solve the issues of aggregation and oxidation of metal salts like polymers and natural ligands $[11,12]$.

Now days, plants are the most commonly used biological agents for synthesizing Cu-nanoparticles. In a study conducted, leaf extracts of Capparis zeylanica was used to reduce copper sulphate (CuSO4) solution to synthesize Cu-NPs. The fabrication of NPs were done for $12 \mathrm{~h}$, size ranged $50-100 \mathrm{~nm}$ having cubical shape. Moreover, significant relation has been established of these synthesized nanoparticles as an antimicrobial agent against various Gram positive and Gram negative bacteria [13]. Figure 3 illustrates some basics of synthesizing NPs from plants.

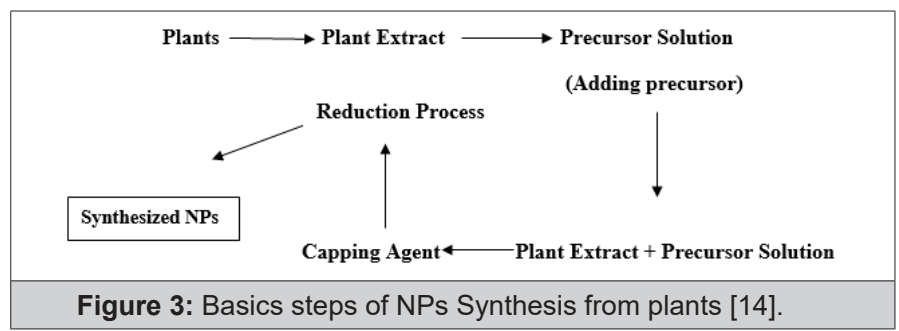

In another study, Syzygium aromaticum (Cloves) extracts was used to synthesize $\mathrm{Cu}$-NPs. The solution of $\mathrm{CuSO} 4$ was reduced with clove extracted solution within $1 \mathrm{hr}$. The optimal size measured to be was of $5-40 \mathrm{~nm}$, having spherical shaped [15]. A report briefed about the synthesis of Cu-NPs from extractions of Ocimum sanctum just within 8-10 minutes. This method showed the rapid characterization and synthesis of $\mathrm{Cu}-\mathrm{NPs}$ which is eco-friendly too [16].

\section{Green Synthesis of Nickle (Ni) Nano-particles}

The literature available today is not sufficient about the Ni-NPs from biological means as compare to physical and chemical method. Green synthesis platform engulf many of the disadvantages of the chemical method as discussed previously i.e. low cost, eco-friendly etc. The main mechanism underlying the synthesis of Ni-NPs is same as of Cu-NPs; the plant extraction material is used as to reduce the metallic salt solution for the synthesis of NPs. The reduction of metallic ions produces various shapes and sizes of NPs using plant as bio templates [17]. The some of the naturally occurring biological molecules are also behaved as efficient reducers in stabilizing and fabricating Ni-NPs such as glucose, sucrose [18-20]. The Ni-NPs biosynthesis is a one step process and produces various stable NPs. The production rate of Ni-NPs is faster than using microbes as secondary metabolites exists in plants such as aldehydes, flavones, terpenoids etc. These metabolites allow faster reduction of metallic salt solution [21].

A study conducted in Ni-NPs synthesis used the extraction material from Medicago sativa (alfalfa) to reduce aqueous solution of solution of Ni (NO3)2. The method involved is the application of precursor solution with alfalfa solution. The temperature was $60 \mathrm{C}$ for $4 \mathrm{hr}$. The reason for high temperature is that it is very difficult to reduce $\mathrm{Ni}(\mathrm{II})$ into $\mathrm{Ni}(0)$ [22]. In another study $\mathrm{Ni}$ (NO3)2·6H2O 
aqueous solution was used as precursor to synthesize Ni-NPs. The plant extract of Ocimum sanctum was used as reducer and as stabilizer [23]. The micro-organisms are also act as stabilizers and reducers for the synthesis and modification of NPs. The potent candidates includes bacteria, yeast and fungi (Figure 2). It is noteworthy that the rate of production of NPs by microbes is slower than plant extracts as of easy processing and handling. Basically, the method involving microbes to synthesize NPs are to culture them first in broth following incubation for specific time and duration on a rotator shaker. Then centrifugation at optimum rpm done and the supernatant is taken to fabricate of Ni-NPs. The incubation for bacteria is done at $30 \mathrm{C}$ at $200 \mathrm{rpm}$ for $120 \mathrm{hr}$, while for fungi $25 \mathrm{C}$ is preferred at $150 \mathrm{rpm}$ for 5 days [24,25].

\section{Antimicrobial Efficacy of $\mathrm{Cu}$ and Ni Nano-particles}

Some studies to test antimicrobial efficacies of $\mathrm{Cu}$ and $\mathrm{Ni}$ nanoparticles also have done. A study measured response of $\mathrm{Cu}$ NPs against Gram positive and Gram negative bacteria i.e. ( $S$. aureus and E. coli respectively) [26]. In another study Datura meta plant extract was used to reduce $\mathrm{CuSO} 4$ solution to produce $\mathrm{Cu}$ NPs, further testing revealed that these NPs have efficient activity against the microbes as compare with the Chloramphenicaol, suggesting as a potent anti-agent [27]. While a study showed that Cu-NPs have significant response against Gram positive and Gram negative bacteria but Gram negative was found to be more sensitive against $\mathrm{Cu}$-NPs which reasoned to be as due to difference in their cell wall composition [28].

$\mathrm{Cu}$-NPs also found as an excellent antimicrobial in another report against bacteria and as well as fungus [29]. The papaya extract used to synthesize Cu-NPs, also tested for antimicrobial effect and showed significant inhibitory effect against E. coli, Klebshiella pneumonia, Pseudomonas aeruginosa and Salmonella typhi. The response to fungus was also responsive; the most prominent effect was found against Fusarium culmorum, then against Fusarium oxysporum and Fusarium graminearum respectively [30]. In similar fashion, Ni-NPs are also showed significant antibacterial activity. According to a study, Ni-NPs are predominant in antibacterial effect against Klebsiella pneumoniae and Proteus vulgaris [31]. The NiNPs synthesized from of Dioscorea (Elephant Yam) extracts treated with nickel sulphate aqueous solution showed antimicrobial activity against four bacterial species tested through disc diffusion method. The strains were S. aureus, B. cereus (Gram-positive) and K. pneumonia, E. coli (Gram-negative). The most effective activity of Ni-NPs were against S. aureus and less effective against $K$. pneumonia [32].

\section{Conclusion}

The nano-particles have become the field of significant interest as it approaches towards many scientific disciplines and establish relationship. The need of time and allocated resources available today also demands for more effective and promising areas to search and develop new potential agents serve as to tackle and limit the danger of AMR. Moreover, it can easily understandable that the plant extraction is much faster and eco-friendly than microbial reduction characteristics because of naturally present secondary metabolites and their derivatives in plants. However, reports showed significant antibacterial activity of $\mathrm{Cu}-\mathrm{NPs}$ and Ni-NPs in parallel fashion to already discovered gold and silver nanoparticles, suggesting $\mathrm{Cu}$ and $\mathrm{Ni}$ as potential agents that further needs to be explored. Because of the lack of knowledge about $\mathrm{Cu}$ and most prominently to Ni there is also a gap in building specific relationship in nano-particles and also to their integrated working fashion. So, we suggest further work and attention should be given to discover hidden facts about $\mathrm{Cu}$ and $\mathrm{Ni}$ nano-particles and hence a comprehensive picture would be established using Green synthesis.

\section{References}

1. Farias CB, Ferreira Silva A, Diniz Rufino R, Moura Luna J, Gomes Souza JE, et al. (2014) Synthesis of silver nanoparticles using a biosurfactant produced in low-cost medium as stabilizing agent. Electronic Journal of Biotechnology 17(3): 122-125.

2. Kaviya S, Santhanalakshmi J, Viswanathan B (2011) Green synthesis of silver nanoparticles using Polyalthia longifolia leaf extract along with D-sorbitol: study of antibacterial activity. Journal of nanotechnology.

3. Hewakuruppu YL, Dombrovsky LA, Chen C, Timchenko V, Jiang X, et al. (2013) Plasmonic "pump-probe" method to study semi-transparent nanofluids. Appl Opt 52(24): 6041-6050.

4. Guozhong C (2004) Nanostructures and nanomaterials: synthesis, properties and applications. World scientific 2: 596.

5. Singh J, Dutta T, Kim KH, Rawat M, Samddar P, et al. (2018) 'Green'synthesis of metals and their oxide nanoparticles: applications for environmental remediation. Journal of nanobiotechnology 16(1): 84 .

6. Raveendran P, Fu J, Wallen SL (2003) Completely "green" synthesis and stabilization of metal nanoparticles. J Am Chem Soc 25(46): 1394013941.

7. Iravani S, Korbekandi H, Mirmohammadi SV, Zolfaghari B (2014) Synthesis of silver nanoparticles: chemical, physical and biological methods. Res Pharm Sci 9(6): 385-406.

8. Mandal D, Bolander ME, Mukhopadhyay D, Sarkar G, Mukherjee P (2006) The use of microorganisms for the formation of metal nanoparticles and their application. Appl Microbiol Biotechnol 69(5): 485-492.

9. Thakkar KN, Mhatre SS, Parikh RY (2010) Biological synthesis of metallic nanoparticles. Nanomedicine: nanotechnology, biology and medicine 6(2): 257-262.

10. Pedersen DB, Wang S, Liang SH (2008) Charge-Transfer-Driven Diffusion Processes in $\mathrm{Cu} @ \mathrm{Cu}$-Oxide Core- Shell Nanoparticles: Oxidation of $3.0 \pm 0.3 \mathrm{~nm}$ Diameter Copper Nanoparticles. The Journal of Physical Chemistry C 112(24): 8819-8826.

11. Crooks RM, Zhao M, Sun L, Chechik V, Yeung LK (2001) Dendrimer-encapsulated metal nanoparticles: synthesis, characterization, and applications to catalysis. Acc Chem Res 34(3): 181-190.

12. Balogh L, Tomalia DA (1998) Poly (amidoamine) dendrimer-templated nanocomposites. 1. Synthesis of zerovalent copper nanoclusters. Journal of the American Chemical Society 120(29): 7355-7356.

13. Saranyaadevi K, Subha V, Ravindran RE, Renganathan S (2014) Synthesis and characterization of copper nanoparticle using Capparis zeylanica leaf extract. Int J Chem Tech Res 6(10): 4533-4454.

14. Rafique M, Shaikh AJ, Rasheed R, Tahir MB, Bakhat HF, et al. (2017) A review on synthesis, characterization and applications of copper nanoparticles using green method. Nano 12(04): 1750043.

15. Subhankari I, Nayak PL (2013) Synthesis of copper nanoparticles using Syzygium aromaticum (Cloves) aqueous extract by using green chemistry. World J Nano Sci Technol 2(1): 14-17.

16. Kulkarni VD, Kulkarni PS (2013) Green synthesis of copper nanoparticles using Ocimum sanctum leaf extract. Int J Chem Stud 1(3): 1-4. 
17. Kar A, Ray AK (2014) Synthesis of nano-spherical nickel by templating hibiscus flower petals. Journal of Nanoscience and Nanotechnology 2(2): 17-20.

18. Raj K, Viswanathan B (2011) Synthesis of nickel nanoparticles with fcc and hcp crystal structures.

19. Vaseem M, Tripathy N, Khang G, Hahn YB (2013) Green chemistry of glucose-capped ferromagnetic hcp-nickel nanoparticles and their reduced toxicity. RSC Advances 3(25): 9698-9704.

20. Dutta A, Dolui SK (2011) Tannic acid assisted one step synthesis route for stable colloidal dispersion of nickel nanostructures. Applied Surface Science 257(15): 6889-6896.

21. Mittal A K, Chisti Y, Banerjee UC (2013) Synthesis of metallic nanoparticles using plant extracts. Biotechnol Adv 31(2): 346-356.

22. Chen H, Wang J, Huang D, Chen X, Zhu J, et al. (2014) Plant-mediated synthesis of size-controllable Ni nanoparticles with alfalfa extract. Materials Letters 122: 166-169.

23. Pandian CJ, Palanivel R, Dhananasekaran S (2015) Green synthesis of nickel nanoparticles using Ocimum sanctum and their application in dye and pollutant adsorption. Chinese journal of Chemical engineering 23(8): 1307-1315.

24. Salvadori MR, Nascimento CAO, Corrêa B (2014) Nickel oxide nanoparticles film produced by dead biomass of filamentous fungus. Sci Rep 4: 6404.

25. Sathyavathi S, Manjula A, Rajendhran J, Gunasekaran P (2014) Extracellular synthesis and characterization of nickel oxide nanoparticles from Microbacterium sp. MRS-1 towards bioremediation of nickel electroplating industrial effluent. Bioresour Technol 165: 270-273.
26. Sampath M, Vijayan R, Tamilarasu E, Tamilselvan A, Sengottuvelan B (2014) Green synthesis of novel jasmine bud-shaped copper nanoparticles. Journal of Nanotechnology p. 7.

27. Parikh P, Zala D, Makwana B (2014) Biosynthesis of copper nanoparticles and their antimicrobial activity. Inst Post Studies Res KSV Uni p. 1-15.

28. Manikandan A, Sathiyabama M (2015) Green synthesis of copper-chitosan nanoparticles and study of its antibacterial activity. J Nanomed Nanotechnol 6(1): 1.

29. Jayandran M, Haneefa MM, Balasubramanian V (2015) Green synthesis of copper nanoparticles using natural reducer and stabilizer and an evaluation of antimicrobial activity. J Chem Pharm Res 7(2): 251-259.

30. Shende S, Ingle AP, Gade A, Rai M (2015) Green synthesis of copper nanoparticles by Citrus medica Linn.(Idilimbu) juice and its antimicrobial activity. World J Microbiol Biotechnol 31(6): 865-873.

31. Sudhasree S, Shakila Banu A, Brindha P, Kurian GA (2014) Synthesis of nickel nanoparticles by chemical and green route and their comparison in respect to biological effect and toxicity. Toxicological \& Environmental Chemistry 96(5): 743-754.

32. Helen SM, Rani MHE (2015) Characterization and antimicrobial study of nickel nanoparticles synthesized from dioscorea (Elephant Yam) by green route. International Journal of Science and Research 4(11): 216219. 